\title{
Appropriate Use Criteria for Somatostatin Receptor PET Imaging in Neuroendocrine Tumors
}

\author{
Thomas A. Hope ${ }^{1,2}$, Emily K. Bergsland ${ }^{3,4}$, Murat Fani Bozkurt ${ }^{5}$, Michael Graham ${ }^{1}$, Anthony P. Heaney ${ }^{6}$, \\ Ken Herrmann ${ }^{5}$, James R. Howe ${ }^{4,7}$, Matthew H. Kulke ${ }^{3,4,8}$, Pamela L. Kunz ${ }^{3,4,8}$, Josh Mailman ${ }^{9}$, Lawrence May ${ }^{10}$, \\ David C. Metz ${ }^{4,11}$, Corina Millo ${ }^{1}$, Sue O’Dorisio ${ }^{1,3,4}$, Diane L. Reidy-Lagunes ${ }^{3,4}$, Michael C. Soulen ${ }^{4,12}$, \\ and Jonathan R. Strosberg 3,4
}

\begin{abstract}
${ }^{1}$ Society of Nuclear Medicine and Molecular Imaging, Reston, Virginia; ${ }^{2}$ American College of Radiology, Reston, Virginia; ${ }^{3}$ American Society of Clinical Oncology, Alexandria, Virginia; ${ }^{4}$ North American Neuroendocrine Tumor Society, Albany, New York; ${ }^{5}$ European Association of Nuclear Medicine, Vienna, Austria; ${ }^{6}$ Endocrine Society, Washington, DC; ${ }^{7}$ Society of Surgical Oncology, Rosemont, Illinois; ${ }^{8}$ National Comprehensive Cancer Network, Fort Washington, Pennsylvania; ${ }^{9}$ NorCal CarciNET, Ripon, California; ${ }^{10}$ American College of Physicians, Washington, DC; ${ }^{11}$ American Gastroenterological Association, Bethesda, Maryland; and ${ }^{12}$ Society of Interventional Oncology, Washington, DC
\end{abstract}

\section{EXECUTIVE SUMMARY}

Somatostatin receptor (SSTR) PET has demonstrated a significant improvement over conventional imaging $(\mathrm{CI})$ in patients with neuroendocrine tumors (NETs). SSTR PET should replace ${ }^{111}$ In-pentetreotide scintigraphy (OctreoScan; Mallinckrodt) in all indications in which the latter is currently being used. These appropriate use criteria (AUC) are intended to aid referring medical practitioners in the appropriate use of SSTR PET for imaging of patients with NETs. The indications were evaluated in welldifferentiated NETs. Of the 12 clinical scenarios evaluated, 9 were graded as appropriate: initial staging after the histologic diagnosis of NET, evaluation of an unknown primary, evaluation of a mass suggestive of NET not amenable to endoscopic or percutaneous biopsy, staging of NET before planned surgery, monitoring of NET seen predominantly on SSTR PET, evaluation of patients with biochemical evidence and symptoms of a NET, evaluation of patients with biochemical evidence of a NET without evidence on CI or a prior histologic diagnosis, restaging at time of clinical or laboratory progression without progression on $\mathrm{CI}$, and new indeterminate lesion on CI with unclear progression. Representatives from the Society of Nuclear Medicine and Molecular Imaging (SNMMI), the American College of Radiology (ACR), the American Society of Clinical Oncology (ASCO), the North American Neuroendocrine Tumor Society (NANETS), the European Association of Nuclear Medicine (EANM), the Endocrine Society, the Society of Surgical Oncology, the National Comprehensive Cancer Network (NCCN), the American College of Physicians (ACP), the American Gastroenterological Association (AGA), and the World Conference on Interventional Oncology (WCIO) assembled under the auspices of an autonomous workgroup to develop the following AUC.

Received Sep. 14, 2017; revision accepted Oct. 6, 2017.

For correspondence or reprints contact: Thomas A. Hope, Department of Radiology and Biomedical Imaging, University of California, San Francisco, 505 Parnassus Ave., San Francisco, CA 94143-0628.

E-mail: thomas.hope@ucsf.edu

Published online Oct. 12, 2017.

COPYRIGHT (C) 2018 by the Society of Nuclear Medicine and Molecular Imaging. DOI: 10.2967/jnumed.117.202275

\section{INTRODUCTION}

NETs

NETs are relatively rare and encompass a heterogeneous group of tumors with an incidence of approximately 7.0 in $100,000(1,2)$, although it is increasing. The most common type is gastroenteropancreatic NETs, which are broken down by sites of origin into gastric, pancreatic, small bowel, colorectal, and those of unknown origin. In addition to gastroenteropancreatic NETs, there are many subtypes of NETs, including pheochromocytomas, paragangliomas, medullary thyroid cancer, Merkel cell cancer, and bronchial carcinoids. Given the lack of evidence in other disease subtypes, these AUC will focus on the role of SSTR PET in well-differentiated gastroenteropancreatic NETs. The belief is that SSTR PET will be valuable in many SSTRpositive diseases beyond gastroenteropancreatic NETs, although these other diseases are not covered in the clinical scenarios in this document.

\section{SSTR}

Somatostatin is a naturally occurring hormone that acts by binding to SSTR, a receptor that is overexpressed on most NETs. There are 5 predominant subtypes of SSTR, type 2 being the most commonly expressed in NETs (3). Somatostatin analogs (SSAs) such as octreotide and lanreotide exert their therapeutic effects by activating SSTRs, which slow tumor growth and inhibit tumorassociated hormone secretion. The presence of SSTRs can be imaged by labeling SSAs with a radionuclide, which was originally performed with octreotide, an octapeptide SSA (4-6). ${ }^{111}$ Inpentetreotide (OctreoScan) was the standard imaging modality for staging and characterizing NETs before SSTR PET.

\section{SSTR PET}

Newer imaging agents targeting SSTR labeled with ${ }^{68} \mathrm{Ga}$ have subsequently been developed, namely, DOTATATE and DOTATOC (7). ${ }^{68} \mathrm{Ga}$-DOTATATE (NETSPOT; Advanced Accelerator Applications) is currently approved by the Food and Drug Administration. A New Drug Application for ${ }^{68} \mathrm{Ga}$-DOTATOC is being developed by the University of Iowa. These agents have several benefits over ${ }^{111} \mathrm{In}$-pentetreotide, including improved detection sensitivity, improved patient convenience due to the 2-h 
length of the study, decreased radiation dose, decreased biliary excretion due to earlier imaging after radiotracer administration, and the ability to quantify uptake. This AUC document focuses on ${ }^{68} \mathrm{Ga}$-DOTATATE and ${ }^{68} \mathrm{Ga}$-DOTATOC, which are collectively referred to as SSTR PET. Few head-to-head data are available that compare different SSTR PET agents, but no relevant differences have been demonstrated between the two agents when used for imaging $(8,9)$. In general, the workgroup agreed that for all indications for which ${ }^{111}$ In-pentetreotide is used, it should be replaced with SSTR PET.

\section{Safety and Dosimetry of SSTR PET}

Human dosimetry data for ${ }^{68} \mathrm{Ga}$-DOTATATE and ${ }^{68} \mathrm{Ga}$ DOTATOC have been reported $(10,11)$, and the estimated totalbody radiation dose is $4.8 \mathrm{mSv}$ for ${ }^{68} \mathrm{Ga}$-DOTATATE and $4.3 \mathrm{mSv}$ for ${ }^{68} \mathrm{Ga}$-DOTATOC for a $185-\mathrm{MBq}(5-\mathrm{mCi})$ administration (Table 1). No adverse events have been reported in association with the administration of SSTR PET agents (12).

\section{Use of Intravenous Contrast with SSTR PET}

Standard PET/CT has frequently been performed without the administration of intravenous contrast. The use of intravenous contrast has been shown to increase the detection rate of liver metastases for ${ }^{18}$ F-FDG PET as well as for SSTR PET $(13,14)$. Contrast can also help with the detection of small-bowel primaries (15). Given the importance of contrast-enhanced imaging studies, we strongly recommend that all SSTR PET studies be performed with intravenous contrast whenever possible. This not only improves the diagnostic accuracy of the imaging study but also prevents the need for additional contrast-enhanced studies in the same patient.

\section{Role of PET/MRI Versus PET/CT}

PET/MRI is a simultaneous modality that allows for PET and MRI to be acquired together. In patients with liver-predominant NETs, this allows improved liver imaging with MRI in conjunction with SSTR PET. Studies have shown that PET/MRI provides improved staging of liver metastases $(16,17)$, but, more important, it allows for the acquisition of liver imaging with the same CI modality as used for monitoring at other times. This is important, as the imaging technique can change the appearance of liver metastases independently of their progression, and therefore a consistent imaging technique needs to be maintained across time. $\mathrm{PET} / \mathrm{CT}$, on the other hand, is superior for patients with mesenteric, osseous, and pulmonary disease. In both PET/MRI and PET/CT, incorporation of contrast-enhanced cross-sectional imaging is encouraged.

\section{Role of SSTR PET in Pediatric Populations}

SSTR PET is safe in infants, children, and young adults. The dose should be adjusted to the patient's weight, with the recommended dose being $2 \mathrm{MBq}(0.054 \mathrm{mCi}) / \mathrm{kg}$ of body weight up to $200 \mathrm{MBq}(5.4 \mathrm{mCi})$ (18). SSTR PET is the recommended functional imaging modality for pediatric NETs and is also recommended for assessing neuroblastoma, paraganglioma, and pheochromocytoma, especially in the setting of metaiodobenzylguanidine-negative disease $(19,20)$. Meningiomas occurring in children and adolescents with neurofibromatosis type 2 express SSTRs and are visualized on SSTR PET. Pediatric indications are not addressed in the clinical scenarios.

\section{Considerations of Tumor Grade and Imaging Modality}

NETs vary in tumor aggressiveness, and tumors are categorized by histologic evaluation. Precise rules for classification vary by tumor site or origin. Gastroenteropancreatic NETs are typically classified on the basis of the Ki-67 proliferation index or the mitotic count (Table 2) (21). Well-differentiated (G1 and G2) NETs are relatively indolent, with a prognosis measured in years even in the face of metastatic disease. High-grade (G3) poorly differentiated neuroendocrine carcinomas (NECs) are typically much more aggressive and nearly always metastatic at diagnosis. Tumors in the recently identified category of well-differentiated G3 NETs are thought to harbor an intermediate prognosis (closer to traditional well-differentiated NETs) (22).

Unresectable well-differentiated NETs of all sites are often treated with liver-directed therapy (e.g., ablation, bland embolization, chemotherapy, or radioembolization), SSAs, or everolimus $(23,24)$. Sunitinib is reserved for patients with advanced pancreatic NETs; temozolomide- or streptozocin-based chemotherapy is also typically reserved for this population (23). Poorly differentiated NECs (e.g., large cell and small cell subtypes) are typically treated with first-line platinum-based chemotherapy or with salvage therapy consisting of several other chemotherapy regimens (i.e., selected from the small cell lung carcinoma armamentarium or regimens commonly used for colorectal cancer if arising in the gastrointestinal tract). An important consideration is that, although data from a randomized trial recently confirmed the value of peptide receptor radionuclide therapy (PRRT) in well-differentiated

TABLE 1

Dosimetry for ${ }^{68} \mathrm{Ga}$-DOTATATE and ${ }^{68} \mathrm{Ga}$-DOTATOC

\begin{tabular}{|c|c|c|c|}
\hline Parameter & ${ }^{68} \mathrm{Ga}-\mathrm{DOTATATE}(10)$ & ${ }^{68} \mathrm{Ga}-\mathrm{DOTATOC}(11)$ & ${ }^{18} \mathrm{~F}-\mathrm{FDG}(56)$ \\
\hline \multicolumn{4}{|l|}{ Organ dose (mSv/MBq) } \\
\hline Kidneys & $9.2 \mathrm{E}-02$ & $2.2 \mathrm{E}-01$ & 1.7E-02 \\
\hline Liver & $4.5 \mathrm{E}-02$ & 7.4E-02 & $2.1 \mathrm{E}-02$ \\
\hline Spleen & $2.8 \mathrm{E}-01$ & $2.4 \mathrm{E}-01$ & $1.1 \mathrm{E}-02$ \\
\hline Bladder wall & 1.3E-01 & $7.0 \mathrm{E}-02$ & 1.3E-01 \\
\hline Effective dose (mSv/MBq) & $2.6 \mathrm{E}-02$ & $2.3 E-02$ & $1.9 \mathrm{E}-02$ \\
\hline \multicolumn{4}{|l|}{ Typical injected activity } \\
\hline $\mathrm{MBq}$ & 185 & 185 & 370 \\
\hline $\mathrm{mCi}$ & 5 & 5 & 10 \\
\hline Estimated effective dose per scan (mSv) & 4.8 & 4.3 & 7.0 \\
\hline
\end{tabular}


TABLE 2

Classification of Gastroenteropancreatic NETs (21)

\begin{tabular}{clcccc}
\hline Differentiation & \multicolumn{1}{c}{ Grade } & Ki-67 index & Proliferative rate & SSTR PET positivity \\
\hline Well differentiated & Low grade (G1) & $<3 \%$ & $<2$ mitoses/10 hpf & +++ \\
& Intermediate grade (G2) & $3 \%-20 \%$ & $2-20$ mitoses $/ 10 \mathrm{hpf}$ & ++ \\
Poorly differentiated & High grade (G3) & $>20 \%$ & $>20$ mitoses/20 hpf & Variable* \\
\hline
\end{tabular}

hpf $=$ high-power field.

*In high-grade NETs, SSTR positivity is variable, and frequently ${ }^{18}$ F-FDG PET performs better as an imaging study in patients with these NETs. SSTR PET results may be positive for well-differentiated G3 tumors, and imaging may be helpful in finding patients who are candidates for PRRT.

NETs arising in the midgut, the use of SSTR PET is less clear in high-grade NECs.

The indications and their appropriateness reviewed in this article bundle grade 1 and grade 2 NETs into one group. The exception to this may be well-differentiated grade 3 NETs, for which optimal treatment is unclear. Patients with these tumors may be candidates for PRRT if they have high expression on SSTR PET; SSTR PET may therefore be helpful in selecting patients for this therapy. Typically, high-grade NECs have lower SSTR expression, as evidenced by less tracer uptake on SSTR PET, and are better imaged with ${ }^{18}$ F-FDG PET (25). Furthermore, significant tumor heterogeneity can occur in patients, with the coexistence of both well-differentiated and poorly differentiated tumors; in this case, a combination of ${ }^{18} \mathrm{~F}-\mathrm{FDG}$ and SSTR PET can be helpful in characterizing disease $(26,27)$.

\section{Understanding Stage Migration When Using SSTR PET}

Several studies indicate that SSTR PET imaging is superior to SSTR scintigraphy or CI (e.g., CT or MRI). For example, SSTR PET can locate the primary tumor site and often demonstrates additional lesions not captured by CI, resulting in better staging that results in clinically relevant changes in management in about one third of patients (28). However, it is important to recognize that identification of more extensive disease may not always have an impact on clinical management and may increase patient and provider anxiety by demonstrating more disease burden than previously visualized with conventional testing. As with any other novel imaging modality, it is important for physicians and patients to realize that direct comparisons between SSTR PET and other imaging tests are not equivalent, and what appears to be disease progression on the first SSTR PET study may simply represent more accurate staging, disease progression being confirmed only by comparing like scans over time.

\section{METHODOLOGY}

\section{Workgroup Selection}

The experts of the AUC workgroup were convened by SNMMI to represent a multidisciplinary panel of health-care providers with substantive knowledge of NETs. In addition to SNMMI member representation, international representatives from ASCO, NANETS, and EANM were included in the workgroup. Nine physician members and 1 patient advocate were ultimately selected to participate and contribute to the resulting AUC. A complete list of workgroup participants can be found in Appendix A. Appendix B is a summary of definitions of terms and acronyms, and Appendix $\mathrm{C}$ provides the disclosures and conflicts-of-interest statement.

\section{AUC Development}

The process for AUC development was modeled after the RAND/UCLA Appropriateness Method $(29,30)$ and included the development of a list of common scenarios encountered in the management of patients with NETs, a systematic review of evidence related to these scenarios, and the development of an appropriateness score for each scenario using a modified Delphi process. This process strove to adhere to the standards of the Institute of Medicine of the National Academies for developing trustworthy clinical guidance (31). The process included a systematic synthesis of available evidence, individual and group ratings of the scenarios using a formal consensus process, and AUC recommendations based on final group ratings and discussions. Development of these AUC based on traditional outcome measures would have been optimal, but the literature review did not return significant numbers of articles with this information.

\section{Scope and Development of Clinical Scenarios (or Indications)}

To begin this process, the workgroup discussed various potential clinical scenarios for which the use of SSTR PET might be considered. The scope of this workgroup was to focus on the appropriate use of SSTR PET specifically for the diagnosis and management of NETs. For all scenarios, the relevant populations were male and female NET patients of any age, race, or geographic location (e.g., rural or urban).

The workgroup identified 12 scenarios for patients with NETs. The scenarios are intended to be as representative of the relevant patient population as possible for development of AUC. The resulting AUC are based on evidence and expert opinion regarding diagnostic accuracy and effects on clinical outcomes and clinical decision making as applied to each scenario. Other factors affecting the AUC recommendations were potential harmincluding long-term harm that may be difficult to capture-costs, availability, and patient preferences.

\section{Systematic Review}

To inform the workgroup, a systematic review of the relevant evidence was commissioned by an independent group, the Pacific Northwest Evidence-Based Practice Center of Oregon Health and Science University (see the supplemental material available at http://jnm.snmjournals.org). The primary purpose of the systematic review was to assess the diagnostic accuracy and comparative effectiveness of SSTR PET in patients with NETs. Two additional metaanalyses were also included in the process $(12,32)$. 
The key research questions used to guide the systematic review were as follows: What is the diagnostic accuracy of SSTR PET compared with ${ }^{111}$ In-pentetreotide, ${ }^{18} \mathrm{~F}$-FDG PET, or CT/MRI for identification of primary NETs, NET metastases, or tumor staging? How does diagnostic accuracy vary according to patient or tumor characteristics (e.g., Ki-67, grade and differentiation, or site of origin)? What is the utility of SSTR PET compared with OctreoScan, ${ }^{18} \mathrm{~F}-\mathrm{FDG}$ PET, or CT/MRI for predicting response to PRRT or SSA therapy? How does predictive utility vary according to patient or tumor characteristics? What are the effects of SSTR PET imaging compared with ${ }^{111} \mathrm{In}$-pentetreotide, ${ }^{18} \mathrm{~F}$-FDG PET, or CT/MRI on clinical decision making? How do effects on clinical decision making vary according to patient or tumor characteristics?

The inclusion and exclusion criteria for papers for this review were based on the study parameters established by the workgroup, using the PICOTS (population, intervention, comparisons, outcomes, timing, and setting) approach. Searches were conducted on the following databases: the Cochrane Central Register of Controlled Trials, the Cochrane Database of Systematic Reviews, and OVID MEDLINE (from 2000 through November 2016). These searches were supplemented by reviewing the reference lists of relevant publications.

Two reviewers independently assessed abstracts and full-text articles for inclusion and rated study quality as defined by the established PICOTS parameters. The quality (based on the risk of bias) of each study was categorized as "good," "fair," or "poor" using the U.S. Preventive Services Task Force criteria for randomized trials and cohort studies (33), Quality Assessment of Diagnostic Accuracy Studies-2 for diagnostic accuracy studies (34), and Assessment of Multiple Systematic Reviews for systematic reviews (35). The strength of overall evidence was graded as high, moderate, low, or very low using methods based on quality of evidence, consistency, directness, precision, and reporting bias.

Literature searches resulted in 635 potentially relevant articles. After a dual review of the abstracts and titles, 237 articles were selected for full-text review and 17 publications were determined to meet the criteria for inclusion in this review.

\section{Rating and Scoring Process}

In developing these AUC for SSTR PET, the workgroup members used the following definition of appropriateness to guide their considerations and group discussions: "The concept of appropriateness, as applied to health care, balances risk and benefit of a treatment, test, or procedure in the context of available resources for an individual patient with specific characteristics" (36).

At the beginning of the process, workgroup members convened at an in-person forum to develop the initial scenarios. On evaluating the evidence summary of the systematic literature review, the workgroup further refined its draft clinical scenarios to ensure their accuracy and facilitate consistent interpretation when scoring each scenario for appropriateness. Using the evidence summary, workgroup members were first asked individually to assess the benefits and risks of SSTR PET for each of the identified scenarios and provide an appropriateness score for each scenario. After deliberate discussion, each member independently provided a second round of scores for each scenario. For each scenario, the mode numeric score was determined and then assigned to the associated appropriate use category. The results of second-round scoring continued to indicate some difference in opinion among members about the appropriateness of certain scenarios. Therefore, the workgroup continued its deliberations and further clarified the criteria for assigning the different scores before conducting a third round of scoring, which reflected a group-level consensus of scores. For this final scoring round, the members were asked to include their expert opinion. All members contributed to the final discussion, and no one was forced into consensus. After the rating process was completed, the final appropriate use ratings were summarized in a format similar to that outlined by the RAND/UCLA Appropriateness Method.

The workgroup scored each scenario as "appropriate," "may be appropriate," or "rarely appropriate" on a scale from 1 to 9. Scores 7-9 indicate that the use of the procedure is appropriate for the specific scenario and is generally considered acceptable. Scores 4-6 indicate that the use of the procedure may be appropriate for the specific scenario. This implies that more research is needed to classify the scenario definitively. Scores 1-3 indicate that the use of the procedure is rarely appropriate for the specific scenario and generally is not considered acceptable.

As stated by other societies that develop AUC, the division of these scores into 3 general levels of appropriateness is partially arbitrary, and the numeric designations should be viewed as a continuum. In addition, if there was a difference in clinical opinion for a scenario such that workgroup members could not agree on a common score, that scenario was given a score of 5 to indicate a lack of agreement on appropriateness based on the available literature and the members' collective clinical opinion, indicating the need for additional research.

\section{CLINICAL SCENARIOS AND AUC SCORES}

Clinical scenarios for the use of SSTR PET and final AUC scores in patients with NETs are presented in Table 3. In grading clinical indications, we focused on well-differentiated NETs.

\section{Scenario 1: Initial Staging After Histologic Diagnosis of NETs (Score 9-Appropriate)}

There was consensus that SSTR PET should be used for the staging of patients with NETs. The systematic review clearly demonstrated the superiority of SSTR PET over both CI and SSTR scintigraphy. It is important to consider the type and size of NETs. For example, patients with subcentimeter rectal NETs likely do not require SSTR PET at initial staging, given the extremely low incidence of metastatic disease in these patients.

\section{Scenario 2: Localization of Primary Tumor in Patients with Known Metastatic Disease but Unknown Primary (Score 9-Appropriate)}

Up to $20 \%$ of patients with NETs have unknown primaries after initial workup, and localization of the primary tumor is important, as treatment options vary depending on the origin of the tumor (37). In one prospective study, the primary tumor was found in $38 \%$ of patients who were imaged with SSTR PET (38). In another paper, the primary tumors of 4 of 14 patients with unknown primaries were detected using SSTR PET (39). This was uniformly agreed to be an appropriate indication for SSTR PET.

\section{Scenario 3: Selection of Patients for SSTR-Targeted PRRT (Score 9-Appropriate)}

PRRT is increasingly becoming an important component of the treatment algorithm for patients with NETs. PRRT localizes radiation delivered by radionuclides, typically ${ }^{177} \mathrm{Lu}$ or ${ }^{90} \mathrm{Y}$, to NET cells by internalization after binding to SSTR. The pivotal prospective randomized phase 3 NETTER-1 trial demonstrated significant prolongation of progression-free survival in patients with midgut NETs after treatment with ${ }^{177}$ Lu-DOTATATE 
TABLE 3

Clinical Scenarios for SSTR PET

\begin{tabular}{|c|c|c|c|}
\hline Scenario no. & Description & Appropriateness & Score \\
\hline 1 & Initial staging after histologic diagnosis of NETs & Appropriate & 9 \\
\hline 2 & $\begin{array}{l}\text { Localization of primary tumor in patients with known metastatic } \\
\text { disease but unknown primary }\end{array}$ & Appropriate & 9 \\
\hline 3 & Selection of patients for SSTR-targeted PRRT & Appropriate & 9 \\
\hline 4 & Staging NETs before planned surgery & Appropriate & 8 \\
\hline 5 & $\begin{array}{l}\text { Evaluation of mass suggestive of NET not amenable to endoscopic or } \\
\text { percutaneous biopsy (e.g., ileal lesion, hypervascular pancreatic mass, } \\
\text { mesenteric mass) }\end{array}$ & Appropriate & 8 \\
\hline 6 & Monitoring of NETs seen predominantly on SSTR PET & Appropriate & 8 \\
\hline 7 & $\begin{array}{l}\text { Evaluation of patients with biochemical evidence and symptoms of } \\
\text { NET without evidence on } \mathrm{Cl} \text { and without prior histologic } \\
\text { diagnosis of NET }\end{array}$ & Appropriate & 7 \\
\hline 8 & $\begin{array}{l}\text { Restaging at time of clinical or laboratory progression without } \\
\text { progression on } \mathrm{Cl}\end{array}$ & Appropriate & 7 \\
\hline 9 & New indeterminate lesion on $\mathrm{Cl}$, with unclear progression & Appropriate & 7 \\
\hline 10 & $\begin{array}{l}\text { Restaging of patients with NETs at initial follow-up after resection } \\
\text { with curative intent }\end{array}$ & May be appropriate & 6 \\
\hline 11 & Selection of patients with nonfunctional NETs for SSA treatment & May be appropriate & 6 \\
\hline 12 & $\begin{array}{l}\text { Monitoring in patients with NETs seen on both } \mathrm{Cl} \text { and SSTR PET } \\
\text { with active disease and no clinical evidence of progression }\end{array}$ & May be appropriate & 5 \\
\hline
\end{tabular}

compared with high-dose octreotide (40). For enrollment, the NETTER-1 trial did not use SSTR PET but required patients to have evidence of SSTR expression on ${ }^{111}$ In-pentetreotide based on the Krenning scale (41). Virtually all other single-arm PRRT studies have required uptake on SSTR imaging as an eligibility criterion. The workgroup agreed that SSTR PET can be used in place of ${ }^{111}$ In-pentetreotide for patient selection for PRRT. Uptake on SSTR PET can be predictive of therapeutic response to PRRT (42), and it is likely that SSTR PET will prove to be a more accurate selection tool than ${ }^{111}$ In-pentetreotide for PRRT, although criteria for positive disease have yet to be developed for SSTR PET.

\section{Scenario 4: Staging NETs Before Planned Surgery (Score 8-Appropriate)}

Published series reporting on surgical cytoreduction of NET liver metastases have demonstrated that, although it is not curative, it improved survival compared with historic controls (e.g., all patients with NET metastases from large national databases) (43-47). The conventional wisdom is that surgical debulking "sets the clock back" but does not cure patients; thus, the presence of extrahepatic disease is not necessarily an absolute contraindication. With the development of SSTR PET, more extensive metastatic disease is being detected, and there is no consensus on how to manage patients surgically if extensive nonresectable disease is seen on SSTR PET. If the bulk of metastatic disease is in the liver or abdominal lymph nodes, then surgical intervention may be warranted. In cases with extensive bone, mediastinal, or neck metastases, the benefits of hepatic cytoreduction are less clear, especially in those patients with impaired performance status and higher-grade tumors. Nonetheless, the workgroup agreed that SSTR PET should be used to guide surgical planning and to rule out extensive extraabdominal disease in patients before undergoing hepatic cytoreductive procedures.
Scenario 5: Evaluation of Mass Suggestive of NET Not Amenable to Endoscopic or Percutaneous Biopsy (e.g., lleal Lesion, Hypervascular Pancreatic Mass, Mesenteric Mass) (Score 8-Appropriate)

A major role for SSTR PET is to demonstrate the presence of SSTRs noninvasively. This can help narrow the differential diagnosis of a lesion and therefore help determine the correct treatment algorithm. In the setting in which a biopsy is not easily obtained, either because of technical limitations such as the lack of access to enteroscopy or because of increased risk of invasive biopsy such as a hypervascular lesion or one too close to large vessels, SSTR PET can demonstrate noninvasively that an uncharacterized mass is SSTR-positive and therefore most likely a NET. In addition, other SSTR-positive disease may be revealed that is more amenable to biopsy.

\section{Scenario 6: Monitoring of NETs Seen Predominantly on SSTR PET (Score 8-Appropriate)}

With SSTR PET, we are seeing more disease that is not appreciable on CI. In particular, osseous metastatic disease is frequently underestimated by CI $(39,48)$, and the only way to visualize the extent of disease is using SSTR PET. In these cases, when the extent of disease cannot be reliably visualized on CI, SSTR PET is indicated for routine imaging and follow-up.

\section{Scenario 7: Evaluation of Patients with Biochemical Evidence and Symptoms of NET Without Evidence of It on $\mathrm{Cl}$ and Without Prior Histologic Diagnosis of NET (Score 7-Appropriate)}

This indication resulted in significant disagreement within the workgroup. On the one hand, the overall yield of finding a NET in this patient population is low, and SSTR PET may also result in false-positives that could lead to unnecessary additional tests or procedures (12). However, in such a situation, a negative SSTR 
PET result may play an important role, as it could end the diagnostic workup, resulting in a more cost-effective evaluation. Furthermore, on the rare occasion that a study result is positive, further investigation of the lesion may be useful in identifying the tumors that are present.

\section{Scenario 8: Restaging at Time of Clinical or Laboratory Progression Without Progression on $\mathrm{Cl}$ (Score 7-Appropriate)}

There was a concern that in comparison to CI, SSTR PET may demonstrate apparent progression that would be misinterpreted and lead to inappropriate changes in management. Baseline imaging with SSTR PET is essential, since comparison with CI would likely show more disease. Nonetheless, SSTR PET allows better evaluation of disease than does $\mathrm{CI}$, and therefore in the setting of clinical or biochemical progression, it can be important for selecting the appropriate therapy.

\section{Scenario 9: New Indeterminate Lesion on $\mathrm{Cl}$, with Unclear Progression (Score 7-Appropriate)}

SSTR positivity is an important finding for demonstrating that a lesion is in fact a NET; therefore, to characterize a finding on CI, SSTR PET can be used to clarify whether a suspected lesion is a NET and represents true progression or recurrence. In addition, it is possible for NETs to dedifferentiate, changing from welldifferentiated to poorly differentiated NETs over time (49). SSTR PET can be an indirect indicator of grade, and therefore reimaging at the time of progression can provide insight into possible underlying dedifferentiation of a tumor.

\section{Scenario 10: Restaging of Patients with NETs at Initial Follow-up After Resection with Curative Intent (Score 6-May Be Appropriate)}

There was a lack of consensus among the committee for this indication. One concern was that it would lead to overuse of SSTR PET in patients without evidence of disease. Many suggested that a single SSTR PET may be indicated after resection, but the main issue with the indication was the lack of impact on patient management. Visualizing small-volume residual disease after surgical resection is unlikely to change patient management; thus, some felt that it would be more appropriate to wait for biochemical evidence for recurrence or radiologic evidence on CI before performing SSTR PET. If a patient did not undergo SSTR PET before surgical resection, a single SSTR PET examination should be considered to complete staging postoperatively.

\section{Scenario 11: Selection of Patients with Nonfunctional NETs for SSA Treatment (Score 6-May Be Appropriate)}

Although it is very likely that SSTR expression correlates with benefit from SSA treatment, this has not been proven definitively in clinical trials. The CLARINET trial, which demonstrated the antiproliferative activity of lanreotide in gastroenteropancreatic NETs, required evidence of SSTR expression with ${ }^{111}$ In-pentetreotide for enrollment (50). The PROMID study, which evaluated octreotide in midgut NETs, did not require evidence of SSTR expression; however, only $12 \%$ of patients had negative imaging results with ${ }^{111} \mathrm{In}$ pentetreotide (51). Only one study has reported that higher uptake on SSTR PET predicts improved response to SSA therapy (52). Because of the benign side effect profile of SSAs, the workgroup did not reach a consensus that confirmation of SSTR expression is necessary for initiation of treatment with octreotide or lanreotide. The workgroup also noted that in syndromic patients, SSTR analogs should be initiated independently of findings on SSTR PET.

\section{Scenario 12: Monitoring in Patients with NETs Seen on Both $\mathrm{Cl}$ and SSTR PET with Active Disease and No Clinical Evidence of Progression (Score 5-May Be Appropriate)}

The consensus was that if CI can detect metastatic disease, then SSTR PET should not be used for routine imaging. There was a belief that intermittent SSTR PET (once every 2-3 y) may be helpful in evaluating for progression if CI results are stable, although intermittent SSTR PET should not be used in place of CI for routine monitoring of patients.

\section{Summary of Recommendations}

SSTR PET should replace ${ }^{111} \mathrm{In}$-pentetreotide in all indications in which ${ }^{111}$ In-pentetreotide is currently being used. SSTR PET has demonstrated better sensitivity and specificity than CI and ${ }^{111}$ In-pentetreotide. There are specific instances in which SSTR PET is clearly preferred: at initial diagnosis, when selecting patients for PRRT, and for localization of unknown primaries. For patients in whom the tumor is readily seen on CI, SSTR PET is not needed for routine monitoring.

\section{BENEFITS AND HARMS OF IMPLEMENTING THE AUC GUIDANCE}

Some providers have raised the concern that AUC for medical imaging might inappropriately limit access to health-care services (53). For example, several authors of papers included in our metaanalysis suggested that the AUC might lead to denial of reimbursement for needed imaging services because of incomplete AUC or lack of strong evidence for a particular procedure (54). It is hoped that besides providing recommendations for the appropriate use of SSTR PET, this document will demonstrate gaps in the literature and subsequently encourage new investigations to address these gaps.

Integration of AUC into clinical decision support tools can assist health-care providers and offer a way to track comparisons between the AUC model and the payer's reimbursement policy $(54,55)$. Ultimately, this may lead to a more efficient approval process for advanced diagnostic imaging procedures, including radiology and nuclear medicine procedures, saving time and effort for the referring provider and the imaging facility. However, the difficult task of writing AUC for all scenarios and keeping the AUC current remains a large obstacle to the effective use of the clinical decision support model.

\section{QUALIFYING STATEMENTS}

Although a large body of literature focuses on SSTR PET, the workgroup found the medical literature regarding the use of SSTR PET to be limited when rigorous inclusion criteria were applied to the systematic literature review. Because most articles did not use pathology as a correlate to imaging, sensitivity and specificity measurements were often limited. Information was also scarce on the role of SSTR PET in high-grade NECs and other less common subtypes of NETs (e.g., well-differentiated G3 NETs, paragangliomapheochromocytoma). In addition, few data were available on the use of SSTR PET in pediatric populations or on how SSTR PET can be used to predict and evaluate the response to PRRT.

\section{IMPLEMENTATION OF THIS AUC GUIDANCE}

SNMMI has been working with several other medical specialty societies to develop broad-based multidisciplinary clinical guidance 
documents. This collaboration should foster the acceptance and adoption of this guidance by other specialties.

SNMMI has developed a multipronged approach to disseminate the AUC for SSTR PET in NETs to all relevant stakeholdersreferring physicians, nuclear medicine physicians, and patients. The dissemination and implementation tactics will be a mix of outreach and educational activities and will be targeted to each of these audiences.

SNMMI will create detailed case studies for its members and for referring physicians and make them available via online modules and webinars. These cases will cover the appropriate clinical scenarios for the use of SSTR PET, as well as some cases in which the results of SSTR PET are equivocal.

Related resources such as the systematic review supporting the development of these AUC, a list of upcoming education events on the AUC, factsheets, and other didactic materials will be made available on the SNMMI webpage dedicated to the SSTR PET AUC. Live sessions will be held at the SNMMI annual and midwinter meetings, as well as at the relevant societal meetings of referring physicians, to highlight the importance of these AUC.

SNMMI also aims to create a mobile application for the SSTR PET AUC for both Apple and Android platforms. Mobile applications are becoming increasingly popular in the health-care industry and can be used to push updates to all users.

In addition to these activities, SNMMI will undertake patient-focused outreach to provide education on how AUC can play an invaluable role in achieving a more accurate diagnosis.

\section{APPENDIX A: WORKGROUP MEMBERS AND LITERATURE REVIEWERS}

Workgroup members: Thomas A. Hope, MD (chair), University of California, San Francisco, San Francisco, CA (SNMMI/ACR); Emily K. Bergsland, MD, University of California, San Francisco, San Francisco, CA (ASCO/NANETS); Murat Fani Bozkurt, MD, Hacettepe University, Ankara, Turkey (EANM); Michael Graham, $\mathrm{PhD}, \mathrm{MD}$, University of Iowa, Iowa City, IA (SNMMI); Anthony P. Heaney, MD, University of California, Los Angeles, Los Angeles, CA (Endocrine Society); Ken Herrmann, MD, Universitatsklinikum Essen, Essen, Germany (EANM); James R. Howe, MD, University of Iowa, Iowa City, IA (Society of Surgical Oncology/NANETS); Matthew H. Kulke, MD, Dana-Farber Cancer Institute, Boston, MA (ASCO/NANETS/NCCN); Pamela Kunz, MD, Stanford University, Stanford, CA (ASCO/NANETS); Josh Mailman, President, NorCal Carcinet, Ripon, CA (patient advocate); Lawrence May, MD, Los Angeles, CA (ACP); David C. Metz, MD, University of Pennsylvania, Philadelphia, PA (AGA/NANETS); Corina Millo, MD, National Institutes of Health, Bethesda, MD (SNMMI); Sue O'Dorisio, MD, PhD, University of Iowa, Iowa City, IA (SNMMI/ASCO/NANETS); Diane L. Reidy-Lagunes, MD, Memorial Sloan Kettering Cancer Center, New York, NY (ASCO/NANETS); Michael C. Soulen, MD, University of Pennsylvania, Philadelphia, PA (NANETS, WCIO); and Jonathan R. Strosberg, MD, Moffitt Cancer Center, Tampa, FL (ASCO, NANETS).

Literature reviewers: Roger Chou, MD, Oregon Health Sciences University, Portland, OR; Elaine Graham, Oregon Health Sciences University, Portland, OR; Miranda Pappas, Oregon
Health Sciences University, Portland, OR; and Barbara Ray, Oregon Health Sciences University, Portland, OR.

SNMMI staff support: Sukhjeet Ahuja, MD, MPH, Director, Evidence \& Quality Department; Julie Kauffman, Program Manager, Evidence \& Quality Department; and Bonnie Clarke, Director, Clinical Trials Network.

\section{APPENDIX B: DEFINITION OF TERMS AND ACRONYMS}

ACP: American College of Physicians

ACR: American College of Radiology

AGA: American Gastroenterological Association

ASCO: American Society of Clinical Oncology

AUC: appropriate use criteria

CI: conventional imaging (CT, MRI, ultrasound, plain film radiography)

CT: computed tomography (imaging method that uses x-rays to create pictures of cross-sections of the body)

EANM: European Association of Nuclear Medicine

Ki67: marker of cellular proliferation

MRI: magnetic resonance imaging

NANETS: North American Neuroendocrine Tumor Society

NCCN: National Comprehensive Cancer Network

NEC: neuroendocrine carcinoma

NET: neuroendocrine tumor

OctreoScan: ${ }^{111}$ In-pentetreotide scintigraphy

PET: positron emission tomography

PET/CT: combination device that provides detail on both function and anatomy by superimposing the precise location of abnormal metabolic activity (from PET) on a detailed anatomic image (from CT)

PICOTS: population, intervention, comparisons, outcomes, timing, and setting

PRRT: peptide receptor radionuclide therapy

SNMMI: Society of Nuclear Medicine and Molecular Imaging

SSA: somatostatin analog

SSTR: somatostatin receptor

WCIO: World Conference on Interventional Oncology

\section{APPENDIX C: DISCLOSURES AND CONFLICTS OF INTEREST}

SNMMI rigorously attempted to avoid any actual, perceived, or potential conflicts of interest (COIs) that might have arisen as a result of an outside relationship or personal interest on the part of the workgroup members or external reviewers. Workgroup members were required to provide disclosure statements of all relationships that might be perceived as real or potential COIs. These statements were reviewed and discussed by the workgroup chair and SNMMI staff and were updated and reviewed by an objective third party at the beginning of every workgroup meeting 
or teleconference. The disclosures of the workgroup members can be found in Table 1C. A COI was defined as a relationship with industry-including consulting, speaking, research, and nonresearch activities - that exceeds $\$ 5,000$ in funding over the previous or upcoming 12-mo period. In addition, if an external reviewer was either the principal investigator of a study or another key member of the study personnel, that person's participation in the review was considered likely to present a COI. All reviewers were asked about any potential COI. A COI was also considered likely if an external reviewer or workgroup member was either the principal investigator or a key member of a study directly related to the content of this AUC document. All external reviewers were asked about any potential $\mathrm{COI}$.

TABLE 1C

Relationships with Industry and Other Entities

\begin{tabular}{|c|c|}
\hline $\begin{array}{l}\text { Workgroup } \\
\text { member }\end{array}$ & Reported relationships \\
\hline Bergsland, Emily & - None \\
\hline $\begin{array}{l}\text { Bozkurt, Murat } \\
\text { Fani }\end{array}$ & - None \\
\hline Graham, Michael & - None \\
\hline Heaney, Anthony & - None \\
\hline Herrmann, Ken & - None \\
\hline Hope, Thomas & $\begin{array}{l}\text { - GE Healthcare, Speakers Bureau, } \\
\text { PET/MRI }\end{array}$ \\
\hline Howe, James & - None \\
\hline Kulke, Matthew & $\begin{array}{l}\text { - Ipsen, Consulting } \\
\text { - Lexicon, Consulting } \\
\text { - Novartis, Consulting }\end{array}$ \\
\hline Kunz, Pamela & $\begin{array}{l}\text { - Ipsen, Ad Board, NETS } \\
\text { - Lexicon, Ad Board, NETS } \\
\text { - Novartis, Ad Board, NETS }\end{array}$ \\
\hline Mailman, Josh & - None \\
\hline May, Lawrence & $\begin{array}{l}\text { - Ideal Living, Infomercial, Prostate } \\
\text { - Ana Home Care, Director }\end{array}$ \\
\hline Metz, David & $\begin{array}{l}\text { - AAA, Grant, NETS } \\
\text { - Ironwood, Grant, GERD } \\
\text { - Lexicon, Grant, NETS } \\
\text { - Novartis, Consulting, NETS } \\
\text { - Wren Labs, Grant, NETS }\end{array}$ \\
\hline Millo, Corina & - None \\
\hline O’Dorisio, Sue & - None \\
\hline $\begin{array}{l}\text { Reidy-Lagunes, } \\
\text { Diane }\end{array}$ & $\begin{array}{l}\text { - Ipsen, Consulting/Research, NETS } \\
\text { - Novartis, Consulting/Research, NETS }\end{array}$ \\
\hline Soulen, Michael & $\begin{array}{l}\text { - Guerbe Health, Grants/Consulting, } \\
\text { NETS, NEC }\end{array}$ \\
\hline $\begin{array}{l}\text { Strosberg, } \\
\text { Jonathan }\end{array}$ & $\begin{array}{l}\text { - Ipsen, Speakers Bureau } \\
\text { - Lexicon, Consulting } \\
\text { - Novartis, Consulting }\end{array}$ \\
\hline
\end{tabular}

\section{REFERENCES}

1. Hallet J, Law CHL, Cukier M, Saskin R, Liu N, Singh S. Exploring the rising incidence of neuroendocrine tumors: a population-based analysis of epidemiology, metastatic presentation, and outcomes. Cancer. 2015;121:589-597.

2. Dasari A, Shen C, Halperin D, et al. Trends in the incidence, prevalence, and survival outcomes in patients with neuroendocrine tumors in the United States. JAMA Oncol. 2017;3:1335-1342.

3. John M, Meyerhof W, Richter D, et al. Positive somatostatin receptor scintigraphy correlates with the presence of somatostatin receptor subtype 2. Gut. 1996;38:33-39.

4. Krenning EP, Kwekkeboom DJ, Bakker WH, et al. Somatostatin receptor scintigraphy with [ ${ }^{111}$ In-DTPA-D-Phe1]- and [ ${ }^{123}$ I-Tyr3]-octreotide: the Rotterdam experience with more than 1000 patients. Eur J Nucl Med. 1993;20:716-731.

5. Bombardieri E, Ambrosini V, Aktolun C, et al. ${ }^{111}$ In-pentetreotide scintigraphy: procedure guidelines for tumour imaging. Eur J Nucl Med Mol Imaging. 2010; 37:1441-1448.

6. Lamberts SW, Bakker WH, Reubi JC, Krenning EP. Somatostatin-receptor imaging in the localization of endocrine tumors. N Engl J Med. 1990;323:12461249 .

7. Antunes P, Ginj M, Zhang H, et al. Are radiogallium-labelled DOTA-conjugated somatostatin analogues superior to those labelled with other radiometals? Eur $J$ Nucl Med Mol Imaging. 2007;34:982-993.

8. Poeppel TD, Binse I, Petersenn S, et al. ${ }^{68} \mathrm{Ga}$-DOTATOC versus ${ }^{68} \mathrm{Ga}$-DOTATATE PET/CT in functional imaging of neuroendocrine tumors. J Nucl Med. 2011; 52:1864-1870.

9. Velikyan I, Sundin A, Sörensen J, et al. Quantitative and qualitative intrapatient comparison of ${ }^{68} \mathrm{Ga}$-DOTATOC and ${ }^{68} \mathrm{Ga}$-DOTATATE: net uptake rate for accurate quantification. J Nucl Med. 2014;55:204-210.

10. Walker RC, Smith GT, Liu E, Moore B, Clanton J, Stabin M. Measured human dosimetry of ${ }^{68}$ Ga-DOTATATE. J Nucl Med. 2013;54:855-860.

11. Hartmann H, Zöphel K, Freudenberg R, et al. Radiation exposure of patients during ${ }^{68} \mathrm{Ga}$-DOTATOC PET/CT examinations [in German]. Nucl Med (Stuttg). 2009;48:201-207.

12. Graham MM, Gu X, Ginader T, Breheny P, Sunderland JJ. ${ }^{68}$ Ga-DOTATOC imaging of neuroendocrine tumors: a systematic review and metaanalysis. $\mathrm{J} \mathrm{Nucl}$ Med. 2017;58:1452-1458.

13. Badiee S, Franc BL, Webb EM, et al. Role of IV iodinated contrast material in ${ }^{18}$ F-FDG PET/CT of liver metastases. AJR. 2008;191:1436-1439.

14. Ruf J, Heuck F, Schiefer J, et al. Impact of multiphase ${ }^{68} \mathrm{Ga}$-DOTATOC-PET/CT on therapy management in patients with neuroendocrine tumors. Neuroendocrinology. 2010;91:101-109.

15. Schreiter NF, Maurer M, Pape U-F, Hamm B, Brenner W, Froeling V. Detection of neuroendocrine tumours in the small intestines using contrast-enhanced multiphase Ga-68 DOTATOC PET/CT: the potential role of arterial hyperperfusion. Radiol Oncol. 2014;48:120-126.

16. Hope TA, Pampaloni MH, Nakakura E, et al. Simultaneous ${ }^{68}$ Ga-DOTA-TOC PET/MRI with gadoxetate disodium in patients with neuroendocrine tumor. Abdom Imaging. 2015;40:1432-1440.

17. Sawicki LM, Deuschl C, Beiderwellen K, et al. Evaluation of ${ }^{68} \mathrm{Ga}$-DOTATOC PET/MRI for whole-body staging of neuroendocrine tumours in comparison with ${ }^{68}$ Ga-DOTATOC PET/CT. Eur Radiol. 2017;26:3063-3069.

18. NETSPOT (kit for the preparation of gallium Ga 68 dotatate injection), for intravenous use [highlights of prescribing information]. U.S. Food and Drug Administration website. https://www.accessdata.fda.gov/drugsatfda_docs/label/ 2016/208547s000lbl.pdf. Revised June 2016. Accessed November 6, 2017.

19. Kong G, Hofman MS, Murray WK, et al. Initial experience with gallium-68 DOTA-octreotate PET/CT and peptide receptor radionuclide therapy for pediatric patients with refractory metastatic neuroblastoma. J Pediatr Hematol Oncol. 2016;38:87-96.

20. Kroiss A, Putzer D, Uprimny C, et al. Functional imaging in phaeochromocytoma and neuroblastoma with ${ }^{68} \mathrm{Ga}$-DOTA-Tyr 3-octreotide positron emission tomography and ${ }^{123}$ I-metaiodobenzylguanidine. Eur J Nucl Med Mol Imaging. 2011;38:865-873.

21. Klimstra DS, Modlin IR, Coppola D, Lloyd RV, Suster S. The pathologic classification of neuroendocrine tumors: a review of nomenclature, grading, and staging systems. Pancreas. 2010;39:707-712.

22. Raj N, Valentino E, Capanu M, et al. Treatment response and outcomes of grade 3 pancreatic neuroendocrine neoplasms based on morphology: well differentiated versus poorly differentiated. Pancreas. 2017;46:296-301.

23. Raymond E, Dahan L, Raoul J-L, et al. Sunitinib malate for the treatment of pancreatic neuroendocrine tumors. N Engl J Med. 2011;364:501-513.

24. Yao JC, Fazio N, Singh S, et al. Everolimus for the treatment of advanced, non-functional neuroendocrine tumours of the lung or gastrointestinal tract 
(RADIANT-4): a randomised, placebo-controlled, phase 3 study. Lancet. 2016; 387:968-977.

25. Kayani I, Bomanji JB, Groves A, et al. Functional imaging of neuroendocrine tumors with combined PET/CT using ${ }^{68} \mathrm{Ga}$-DOTATATE (DOTA-DPhe1,Tyr3octreotate) and ${ }^{18}$ F-FDG. Cancer. 2008;112:2447-2455.

26. Hofman MS, Hicks RJ. Changing paradigms with molecular imaging of neuroendocrine tumors. Discov Med. 2012;14:71-81.

27. Chan DL, Pavlakis N, Schembri GP, et al. Dual somatostatin receptor/FDG PET/CT imaging in metastatic neuroendocrine tumours: proposal for a novel grading scheme with prognostic significance. Theranostics. 2017;7:11491158 .

28. Barrio M, Czernin J, Fanti S, et al. The impact of somatostatin receptor-directed $\mathrm{PET} / \mathrm{CT}$ on the management of patients with neuroendocrine tumor: a systematic review and meta-analysis. J Nucl Med. 2017;58:756-761.

29. Hendel RC, Patel MR, Allen JM, et al. Appropriate use of cardiovascular technology: 2013 ACCF appropriate use criteria methodology update: a report of the American College of Cardiology Foundation appropriate use criteria task force. J Am Coll Cardiol. 2013;61:1305-1317.

30. Fitch K, Bernstein SJ, Aguilar MD, Burnand B. The RAND/UCLA Appropriateness Method User's Manual. Santa Monica, CA: RAND; 2001.

31. Institute of Medicine of the National Academy. Clinical Practice Guidelines We Can Trust. Washington, DC: National Academies Press; 2011.

32. Deppen SA, Blume J, Bobbey AJ, et al. ${ }^{68} \mathrm{Ga}$-DOTATATE compared with ${ }^{111} \mathrm{In}$ DTPA-octreotide and conventional imaging for pulmonary and gastroenteropancreatic neuroendocrine tumors: a systematic review and meta-analysis. $\mathrm{J} \mathrm{Nucl}$ Med. 2016;57:872-878.

33. U.S. Preventive Services Task Force Procedure Manual. Rockville, MD: Agency for Healthcare Research and Quality; 2008.

34. Whiting PF, Rutjes AWS, Westwood ME, et al. QUADAS-2: a revised tool for the quality assessment of diagnostic accuracy studies. Ann Intern Med. 2011;155: 529-536.

35. Shea BJ, Bouter LM, Peterson J, et al. External validation of a measurement tool to assess systematic reviews (AMSTAR). PLOS ONE. 2007;2:e1350-e1355.

36. Appropriate use criteria (AUC) development process. Society of Nuclear Medicine and Molecular Imaging website. http://www.snmmi.org/ClinicalPractice/ content.aspx? ItemNumber $=15665$. Accessed November 6, 2017.

37. Bellizzi AM. Assigning site of origin in metastatic neuroendocrine neoplasms: a clinically significant application of diagnostic immunohistochemistry. Adv Anat Pathol. 2013;20:285-314.

38. Menda Y, O'Dorisio TM, Howe JR, et al. Localization of unknown primary site with ${ }^{68} \mathrm{Ga}$-DOTATOC PET/CT in patients with metastatic neuroendocrine tumor. J Nucl Med. 2017;58:1054-1057.

39. Sadowski SM, Neychev V, Millo C, et al. Prospective study of ${ }^{68}$ Ga-DOTATATE positron emission tomography/computed tomography for detecting gastroentero-pancreatic neuroendocrine tumors and unknown primary sites. J Clin Oncol. 2016;34:588-596.
40. Strosberg J, El-Haddad G, Wolin E, et al. Phase 3 trial of ${ }^{177}$ Lu-dotatate for midgut neuroendocrine tumors. N Engl J Med. 2017;376:125-135.

41. Krenning EP, Valkema R, Kooij PP, et al. Scintigraphy and radionuclide therapy with [indium-111-labelled-diethyl triamine penta-acetic acid-D-Phe1]-octreotide. Ital J Gastroenterol Hepatol. 1999;31(suppl 2):S219-S223.

42. Kratochwil C, Stefanova M, Mavriopoulou E, et al. SUV of $\left[{ }^{68} \mathrm{Ga}\right]$ DOTATOC$\mathrm{PET} / \mathrm{CT}$ predicts response probability of PRRT in neuroendocrine tumors. Mol Imaging Biol. 2015;17:313-318.

43. Sarmiento JM, Heywood G, Rubin J, Ilstrup DM, Nagorney DM, Que FG. Surgical treatment of neuroendocrine metastases to the liver: a plea for resection to increase survival. J Am Coll Surg. 2003;197:29-37.

44. Glazer ES, Tseng JF, Al-Refaie W, et al. Long-term survival after surgical management of neuroendocrine hepatic metastases. HPB (Oxford). 2010;12:427-433.

45. Mayo SC, de Jong MC, Pulitano C, et al. Surgical management of hepatic neuroendocrine tumor metastasis: results from an international multi-institutional analysis. Ann Surg Oncol. 2010;17:3129-3136.

46. Graff-Baker AN, Sauer DA, Pommier SJ, Pommier RF. Expanded criteria for carcinoid liver debulking: maintaining survival and increasing the number of eligible patients. Surgery. 2014;156:1369-1376.

47. Maxwell JE, Sherman SK, O'Dorisio TM, Bellizzi AM, Howe JR. Liver-directed surgery of neuroendocrine metastases: what is the optimal strategy? Surgery. 2016;159:320-333.

48. Putzer D, Gabriel M, Henninger B, et al. Bone metastases in patients with neuroendocrine tumor: ${ }^{68} \mathrm{Ga}$-DOTA-tyr3-octreotide PET in comparison to CT and bone scintigraphy. $J$ Nucl Med. 2009;50:1214-1221.

49. Tang LH, Untch BR, Reidy DL, et al. Well-differentiated neuroendocrine tumors with a morphologically apparent high-grade component: a pathway distinct from poorly differentiated neuroendocrine carcinomas. Clin Cancer Res. 2016;22:1011-1017.

50. Caplin ME, Pavel M, Ćwikła JB, et al. Lanreotide in metastatic enteropancreatic neuroendocrine tumors. $N$ Engl J Med. 2014;371:224-233.

51. Rinke A, Müller H-H, Schade-Brittinger C, et al. Placebo-controlled, doubleblind, prospective, randomized study on the effect of octreotide LAR in the control of tumor growth in patients with metastatic neuroendocrine midgut tumors: a report from the PROMID Study Group. J Clin Oncol. 2009;27:4656-4663.

52. Koch W, Auernhammer CJ, Geisler J, et al. Treatment with octreotide in patients with well-differentiated neuroendocrine tumors of the ileum: prognostic stratification with Ga-68-DOTA-TATE positron emission tomography. Mol Imaging. 2014;13:1-10.

53. Bettmann MA. The ACR appropriateness criteria: view from the committee chair. J Am Coll Radiol. 2006;3:510-512.

54. Thrall JH. Appropriateness and imaging utilization: "computerized provider order entry and decision support." Acad Radiol. 2014;21:1083-1087.

55. Sistrom CL. In support of the ACR appropriateness criteria. J Am Coll Radiol. 2008;5:630-635.

56. Brix G, Lechel U, Glatting G, et al. Radiation exposure of patients undergoing whole-body dual-modality ${ }^{18}$ F-FDG PET/CT examinations. J Nucl Med. 2005;46: 608-613. 